\title{
Cervical Cancer, Types of Diagnosis and Recent Treatment Methodologies
}

\author{
Anusha Sudulaguntla*1, Jeffin Thomas ${ }^{2}$, Aji KU ${ }^{2}$, Gifty Merin Chacko ${ }^{2}$ and Donamol Abraham ${ }^{2}$ \\ ${ }^{1}$ Department of Pharmacy Practice, Siddhartha Institute of Pharmaceutical Sciences, India \\ ${ }^{2}$ Doctor of Pharmacy, T John College of Pharmacy, India
}

Received: 棒 January 24, 2018; Published: 眥 February 08, 2018

*Corresponding author: Anusha Sudulaguntla, Assistant Professor, Department of Pharmacy Practice, Siddhartha Institute of Pharmaceutical Sciences, Jonnalagadda, Narasaraopeta, Guntur-522601, Andhra Pradesh, India, Tel: 0091-9535509073,

Email: anusha9073@gmail.com

\begin{abstract}
Cervical cancer is one of the most common cancers among women in developing countries. Human papilloma virus (HPV) with sexually transmitted infection is the major causative agent and risk factor for developing cervical cancer. Women aged between " $21-65$ years" are the major risk group for cervical cancer. Increase in incidence and mortality rate due to cervical cancer, led to interventions for preventing and screening of this disease. Pap smear and HPV test are the two major screening techniques. According to National Cancer Institute, HPV vaccines can prevent cancerous and pre-cancerous cases infected by high risk HPV. Recent advancement by targeting specific genes provides new scope in the treatment of cervical cancer.
\end{abstract}

Keywords: Cervical cancer; HPV (human papilloma virus); Pap smear; HPV vaccines

Abbreviations: CDC: Center for Disease Control and Prevention, FIGO: International Federation of Gynecology and Obstetrics, AJCC: American Joint Committee on Cancer, HPV: Human papilloma Virus, CIN: Cervical Intraepithelial Neoplasia, DES: Diethylstilbestrol, LEEP: Loop Electrosurgical Excision Procedure.

\section{Introduction}

In case of women cervical cancer is the second most detected type of cancer [1]. Squamous cell cancer is the major type which accounts about $90 \%$ and 10 to $20 \%$ for Adenocarcinoma [2] According to $\mathrm{CDC}$, there is a high rate of occurrence of cervical cancer in Hispanic women [3]. Based on global cancer statistics in

2012 it was found that 14.1 million new cases of cervical cancer are diagnosed and 8.2 million deaths per year occur in the world [4]. A study done by Dr. Surendra Srinivas Shastri reported that $30 \%$ of global mortality rate of cervical cancer is accounted by Indian Women [5](Figure 1).

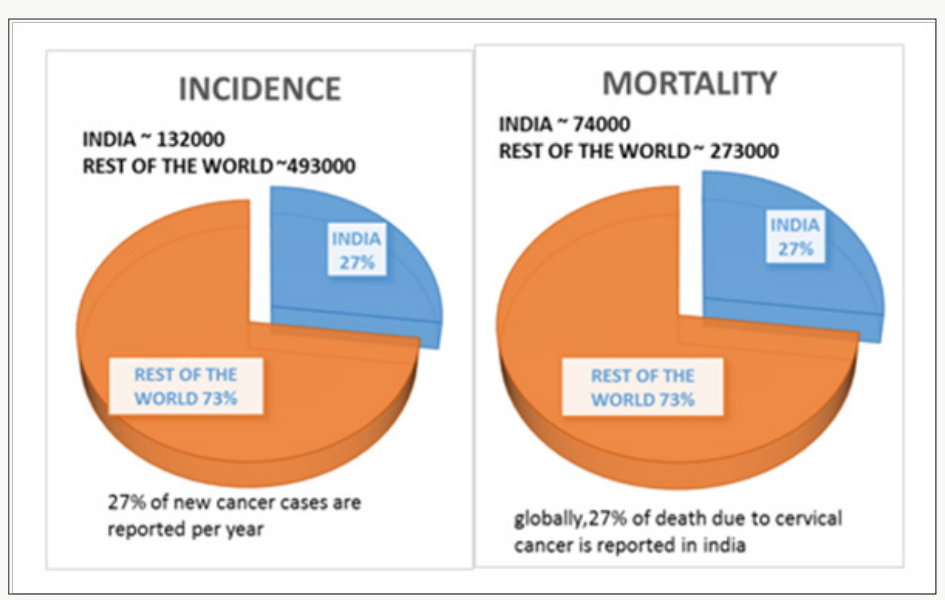

Figure 1: ACCF/ AHA Stages of HF. 


\section{Cervical Cancer Disease Rate}

Women in between 35 to 55 years show more incidence to this type of cancer [6,7]. The main cause is human papilloma virus (HPV) which is accounted for $90 \%$ and other causes involves immune system deficiency, smoking, use of oral contraceptives ,exposure to diethylstilbestrol etc., [8]. HPV 16 and HPV 18 are the high risk HPV types which causes $70 \%$ of cervical cancer [1]. The major signs and symptoms includes: Abnormal bleeding, Pelvic pain, unusual

vaginal discharge, and abnormal menstrual cycle [9]. In case of cervical cancer the main screening procedure is Pap smear [10]. This Pap test helps to diagnose the presence of precancerous and cancerous cells in the cervix [11]. For staging of cervical cancer mainly 2 systems are used, named as FIGO and AJCC. According to FIGO the cervical cancer is staged from 1 to 4 based on clinical examination. The AJCC classifies cervical cancer based on 3 factors:

a.Extend of tumor (T)

b.Lymph node spread (N)

c.Distant spread (M)[12]

\section{Discussion}

\section{Screening of Cervical Cancer}

i. Cervical screening detects cervical cancer and pre-cancerous changes in the cervix.

ii. Cellular abnormalities are tested in laboratories by collecting cells from the surface of cervix.

iii. This screening cannot be done for cancer of reproductive system and sexually transmitted diseases [13].

iv. At the age of 70 two normal results within the past five years is recommended to stop screening.

v. After first exposure of HPV, it develops within 3 to 5 years and leads to cervical cancer [14].

\section{Screening tests are of two types}

a) Pap smear (cytological based screening): Pap smear detects cancerous cells, infection and inflammation like noncancerous conditions. Screening of sample shows the presence of abnormal cells.

b) HPV testing: Detects high-risk HPV types in cervical cells and cell abnormalities. 13-14 high risk HPV types can be identified through sample screening [15].

\section{Types of Screening Results}

Sometimes, the screening results of cervical cancer shows:

A. False-positive result: normal cells are present, but the result may show abnormal cells.
B. False-negative result: abnormal cells may be present but detects as normal.

C. Douching, sexual intercourse, and using vaginal medications or hygiene products before 2 days of test should be avoided to prevent such results. During menstrual period cervical cancer screening should be avoided [15].

In US, at the age of 21 screening can be done, but in some other countries screening begins at the age of 25. Screening below 21 year old girls are not recommended because of high risk of false positive result. [16].

\section{Pap smear and HPV Tests}

Named after Dr.Georgios Papanikolaou, who determined PAP test [17]. Pap test was first introduced in 1940's. In 1999, FDA approved screening methods to detect cancerous cells and first vaccine was developed in 2006 for the prevention of HPV [18]. Cervical cancer can be screened by Pap test for the detection of cancerous and precancerous cells in cervix by scrapping the cells and examines the abnormal cell growth. Collected cells from the cervix will be looked under the microscope to find cancer and precancer cell growth [19]. Pap smear can be done for all the women aged between 21- 65 years, whereas HPV is not advisable for women under the age of 29 years. If PAP and HPV are co tested, I can be done once in 5 years [15].

High risk types of HPV was found by HPV tests. Untreating the HPV types can lead to abnormal cell synthesis. In HPV test, cells from cervix are collected using small soft brush and send to the lab for further investigation or directly from pap sample HPV test sample can be taken. Worldwide about $70 \%$ of cervical cancer was caused by HPV16 and HPV18, high risk HPV types (genotype). HPV test can be used to detect presence of HPV, when Pap test is not clear [20].

\section{Preparation for Pap and HPV Test:}

I. Schedule the date for test, by avoiding the menstruation days. If menstruation begins unexpectedly reschedule the date.

II. 2 days before the test, sexual intercourse should be avoided.

III. Avoid douche 2 days before test.

IV. Avoid vaginal creams and medication inserted in vagina for 48 hrs before test [21].

\section{Procedure}

A speculum, which is a metal or plastic instrument used to keep the vagina open, will be placed inside the vagina to see the cervix properly. Samples of cells and mucus will be scraped from the exocervix using a small spatula. Through the opening of the cervix a small cotton swab or brush will be inserted to take a sample. For hysterectomy patients samples are taken from vaginal cuff (upper part of the vagina). 
Prepared cell samples are examined under a microscope in the laboratory by:

A) The sample are smeared on the slide, sprayed with preservative and send to the laboratory, known as conventional cytology

B) Instead of directly putting the samples on the slide, they will add preservative liquids and send to the lab. This will be spread on to the slide known as liquid based Pap smear.

C) Conventional cytology finds more abnormal cancer cells than liquid based Pap smear. Advantages of liquid based pap smear includes less chance of repeating pap test, and same sample can be for used HPV testing. The disadvantages of this test are, it also detects the non-cancerous cell changes along with cancerous cells and it is more expensive than conventional cytology [19]. The Pap test results show cell changes, usually known as cervical dysplasia [20].

\section{Reporting of Results for Cervical Cancer:}

i. In case of Pap test, the result interprets as normal or abnormal. Similarly HPV test can either be positive (infection with high risk HPV) or negative (no presence of high risk HPV type).

ii. In United States, Bethesda system is the type of standard term used for reporting the results.

iii. Based on Bethesda system, negative is reported for intraepithelial malignancies even if there are no abnormal cells.

iv. Pap test negative report may find non-cancerous cells, like inflammation and common infection [22] (Table 1).

Table 1: Difference between the Pap Smear, A Biopsy and an Hpv Test.

\begin{tabular}{|c|c|c|}
\hline \multicolumn{1}{|c|}{ Pap smear } & Biopsy & HPV test \\
\hline Is used to find the abnormal cells on the cervix. & $\begin{array}{c}\text { Is useful when a cluster of cervix cells is } \\
\text { removed to confirm the results of Pap smear and } \\
\text { to rule out caner. }\end{array}$ & $\begin{array}{c}\text { Is done for detection of DNA of Human } \\
\text { papilloma virus within the cell. }\end{array}$ \\
\hline
\end{tabular}

\section{Who have to Screen for Cervical Cancer?}

Recommendations say that all women between the ages of 21-65 should screen for Pap smear [23,24]. But guidelines recommend women with age 30 or above for starting of cervical cancer screening. It is mainly because of higher risk of cervical cancer in them. 30-49 years aged women should be screened prior to reduce the screening test in their lifetime. Screening risk of CIN2 determines the extent of disease to younger and older women [25].

The latest recommendations suggested by American Cancer Society for screening of cervical cancer are:

A. All women should begin screening at the age of 21 regardless of their sexual activeness [26].

B. Women between the ages of 21-29should have a Pap test every 3 years. They should not be tested for HPV until it is needed after an abnormal Pap test result.

C. Women between the age of 30 and 65 should undergo Pap test and an HPV test every 5 years. This is the preferred approach, but it is also good to have a Pap test alone every 3 years.

D. Women elder than 65 , who have had regular screenings with normal results need not be screened again for cervical cancer. But women who have been diagnosed with cervical pre-cancer should continue with the screening.

E. Women who have taken the HPV vaccine should still follow the screening recommendations for their specific age group.

F. Women who are at high risk are needed to be screened for cervical cancer more often. This high risk conditions may include those with exposure to the drug DES, HIV infection, or organ transplant $[25,27]$.

\section{Cervical cancer and pap smear: who is not screened and why?}

Pap smear is the screening test for cervical cancer. Precancerous or cancerous tissues can be detected by this. With a proper diagnosis and treatment cervical cancer could be cured. Regular screening and early detection of this disease can improve the survival rates and reduce the mortality.

WHO guidelines for screening and treatment of preneoplasmic lesions for cervical cancer recommend a screening and treatment program for patients to achieve the goal [28]. But there are some reasons for which women are not screening for cervical cancer.

i. ACCS (advisory committee of cervical screening) in England recommends no screening test for women aged below 25 as it results in real harm to patient like miscarriage and pre term delivery and over treatment [29].

ii. Harlan et al. compared Hispanic women with black and whites; most of the Hispanic women were unknown about the screening of cervical cancer than blacks or whites. It shows the compliance for screening which is related to race. In the same study they compared Hispanics speaking Spanish and those who are speaking English. It is evident that Spanish speaking women has less compliance than English speaking women because of their low knowledge about this.

iii. Lucina et al. [29] studied the effect of acculturation (or foreign born) on cervical cancer screening in Mexican American 
women. It is described that the positive influence of Hispanics familism helps in increasing the effectiveness of cancer control interventions.

iv. In the comparison study of women below and above poverty index in the past 3 years, the former are less likely to have a Pap smear screening. Low educated women (includes minorities and low socio economic status people) also showed a good rate of noncompliance to Pap smear. That means education as well as income influence the compliance to screen. v. Studies showing that, individuals with no usual problems in their health will not screen for cervical cancer.

vi. Menopausal state of women also showed effect on screening compliance. Non-surgical menopause had less compliance than pre menopause or surgical menopause.

vii. Women who have had their uterus and cervix removed by a hysterectomy and have no history of cervical cancer or precancer need not be screened [30-32].

\section{New Screening Algorithm for Cervical Cancer (Figure 2)}

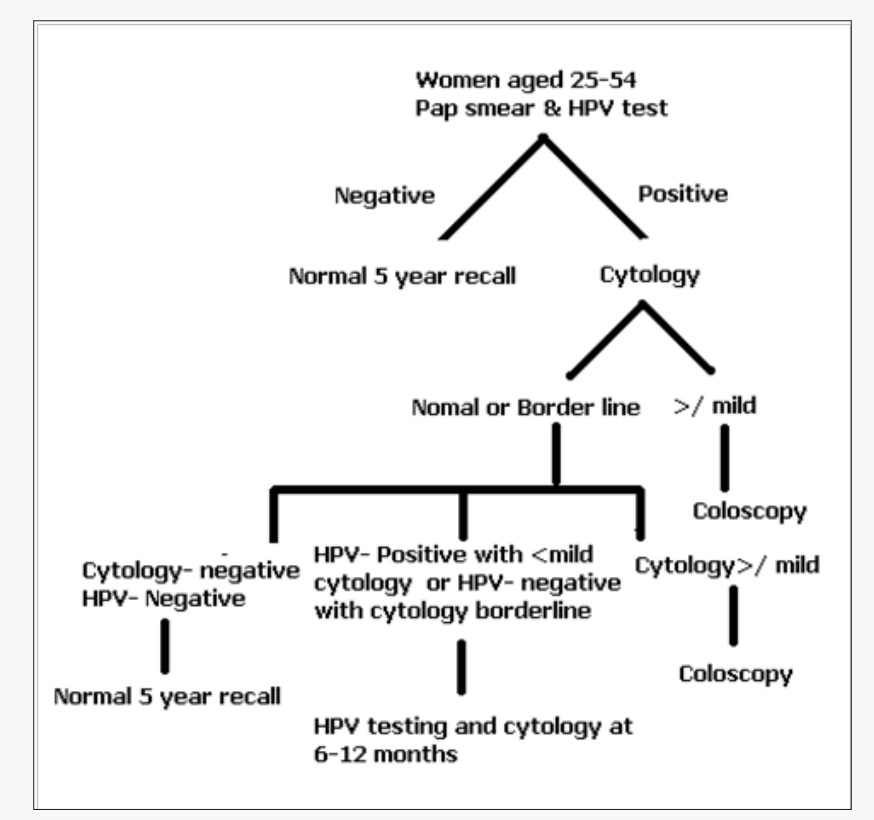

Figure 2: Treatment algorithm for cervical cancer.

\section{Benefits of Pap smear}

a. Pap smear is a rapid test that can be done in few minutes [33].

b. It can detect cervical dysplasia or cervical precancerous changes.

c. Pap test along with HPV test can improve the detection of abnormalities in glandular cells such as cervical adenocarcinoma [34-39].

\section{Limitations of Pap Smear:}

a. Pap test is not efficient to detect Squamous cell abnormalities and cancers.

b. Chance to occur false negative results due to clustering of blood cells on the glass slide.

c. There may be a chance to contagion the sample with other content of cervical specimen like blood, yeast and bacteria which leads to prevent the detection of abnormal cells.

d. Long air contact of the sample may falcificate the cervical cells.

e. By examining the sample, human error may come which affects the detection.

f. Sometimes inadequate collection of sample will be there [3439].

\section{Complications of Pap smear}

After doing Pap test, it's having some complications which are following

Pelvic Discomfort: Little sensation of pelvic discomfort or pain may experience when the doctor takes cervical cells. But, it will recover fast.

Cramping: Abdominal cramping may occur in a mild form. It's a temporary one. If it prolong, analgesics can be taken under the supervision of physician.

Vaginal Bleeding: Scraping cells from the cervix may cause discomfort to the reproductive part which leads to vaginal bleeding. It is in mild form and subside within one day [36]. 


\section{Recent Advancement In Cervical Cancer}

Targeted Therapy: Targeted Therapy inhibits the spreading and further growth of cancerous cells. These works by targeting specific genes or proteins which is found in cancerous cells [40]. It is used along with chemotherapy or radiation therapy [41]. Mainly there are 2 types of targeted therapy: Monoclonal antibodies and small molecule drugs.

Monoclonal antibodies: It blocks the signal activation which is necessary for cell growth, antibody dependent cellular cytotoxicity.

Small molecule drugs: It inhibits cancerous cell multiplication and spreading. One of the examples include, Bevacizumab, it is an angiogenesis blocker and it also belongs to class of monoclonal antibodies which block VEGF-A to prevent angiogenesis. Pazopanib is a targeted drug therapy it act as VEGF and tyrosine kinase inhibitor [42].

Hyperthermia: It includes raising the temperature in the particular area where the tumor is present. It is also indicated along with radiation therapy which avoids the reoccurrence and increase in life span of patients [43].

HPV Vaccine: These are developed in order to prevent infections associated with HPV types [41,44]. Mainly three types of HPV vaccines which are named as Gardasil, Gardasil 9 and Cervarix. The major mechanism by them is to prevent infection due to HPV types 16 and 18. Among these, Gardasil and Gardasil 9 inhibit infection due to other high risk HPV types [45].

LEEP Surgery: In this, through a thin wire hook electrical current is passed, which remove the abnormal cervical tissue found during colposcopy.

Exenteration: This is done when cervical cancer spread to colon, vagina, uterus, rectum along with radiation therapy [44].

Drugs for pre-cancers: It includes conization and cryotherapy which are the standard treatment for CIN. In one study it is found that after administration of an anti-viral drug called Cidofovir, half of the women with CIN treated with this drug got resolved [41].

Adoptive cell Therapy: In this T cells from the patients are removed and in order to enhance the activity either by genetic modification or treated with chemicals. And this is reintroduced in order to improve the anti-cancer response.

Therapeutic vaccines: TVGV-1 vaccine: A phase 2 clinical trial is done for patient with pre cancer which is induced by HPV. VGX3100: A phase 2 clinical trial of this vaccine which mainly target HPV types 16 And 18 [46].

\section{Conclusion}

Incidence and mortality rate of cervical cancer can be reduced by screening techniques like Pap smear and HPV test. Cervical cancer can be prevented by early detection and by treating precancerous conditions. Review on articles based on cervical cancer and screening techniques gives more information about prevention screening of cervical cancer. New Advancement in the field of cervical cancer led a tremendous improvement in prevention.

\section{References}

1. Schiffman M, Castle P, Jeronimo J, Rodriguez A, Wacholder S, et al. (2007) Human papilloma virus and cervical cancer. The Lancet 370(9590): 890907.

2. Michael Frumovitz (2012) Invasive cervical cancer: Epidemiology, risk factors, clinical manifestations, and diagnosis.

3. CDC - Cervical Cancer Rates by Race and Ethnicity. Cdc.gov, USA.

4. CDC - Global Cancer Statistics. Cdc.gov, USA.

5. Vinegar May Reduce Cervical Cancer Risk: ASCO, EverydayHealth.com.

6. Gardasil - Do we need Cervical Cancer Vaccine in India? Slideshare.net, India.

7. Aswathy S, Reshma J, Avani D (2015) Epidemiology of cervical cancer with special focus \& nbsp on India. International Journal of Women's Health: pp. 405-414.

8. Cervical Cancer: Risk Factors (2012) Cancer.Net.

9. Cervical Cancer Symptoms \& Signs, CTCA, Cancercenter.com.

10. Pap Smear (2016) Healthline, USA.

11. Koss L (1993) Cervical (pap) smear: New directions. Cancer 71(S4): 1406-1412.

12. How is cervical cancer staged? Cancer.org.

13. Cancer Screening - About cervical screening (2016) Cancerscreening. govau.

14. Cervical Cancer Screening and Prevention (2016) Clevelandclinicmeded.com.

15. Cervical Cancer Screening - ACOG. Acog.org.

16. Cervical cancer screening. Uptodate.com.

17. Pap Smear. Healthline, USA.

18. Cervical Cancer - Foundation for Women's Cancer. Foundation for Women's Cancer.

19. The Pap (Papanicolaou) test. Cancer.org.

20. Pap Tests and HPV Tests: Screening for Cervical Cancer. NCCC.

21. CDC - What Should I Know About Cervical Cancer Screening? Cdc.gov, USA.

22. Kurman RSolomon D (1991) The Bethesda System for Reporting Cervi$\mathrm{cal} /$ Vaginal Cytologic Diagnoses. International Journal of Gynecological Pathology 10(4): 323-325.

23. Human Papillomavirus (HPV) Genital Warts. Human Papillomavirus (commonly called Genital Warts) Human Papillomavirus (HPV) is a virus that can cause various.Slideplayer.com.

24. Centers of Disease control and Prevention. Cdc.gov, USA.

25. WHO guidelines for screening and treatment of precancerous lesions for cervical cancer prevention, USA.

26. Harvey Simon (2016) Pap smear: Health Guide; the Newyork Times, USA. 
27. HPV and Cervical Cancer: NCCC, India.

28. Tsu V, Jerónimo J (2016) Saving the World's Women from Cervical Cancer. New England Journal of Medicine 374(26): 2509-2511.

29. Al Kalbani M, Price J, Thompson G, Ahmad S, Nagar H (2015) Asian Pacific Journal of Cancer Prevention 16(15): 6557-6559.

30. Harlan L, Bernstein A, Kessler L (1991) Cervical cancer screening: who is not screened and why? Am J Public Health 81(7): 885-890.

31. Cervical cancer screening recommendations 2012: The American society for coloscopy and cervical cancer, USA.

32. Suarez L (1994) Pap smear and mammogram screening in Mexican-American women: the effects of acculturation. American Journal of Public Health 84(5): 742-746.

33. Cervical Cancer Prevention: Toward Cost-Effective Screening, Medscape.

34. San Sebastian, 26 de Marzo de 2010 Laia Alemany Institut Català dOncologia II Congreso socinorte presente y futuro de la prevención. ppt descargar , Slideplayer.es.

35. Cervical Cancer: Practice Essentials, Background, Pathophysiology. Emedicine, medscape.com
36. Uddin RUddin (2017) R Possible Complications of a Pap smear, livestrong.com, USA.

37. Mehta Vasanth V (2009) Pap smear, 75(2): 214-216.

38. Can Cervical Cancer be prevented? American cancer Society, USA.

39. Pap and HPV testing-National Cancer Institute, USA.

40. Target cancer Therapies- National cancer Institute, USA.

41. What's new in cervical cancer research and treatment? Cancerorg, USA.

42. Understanding Targeted Therapy (2013) Cancer.Net, USA.

43. Krishnamurthy A (2015) Recent Advances in Cervical Cancer Avid Science, USA.

44. Cervical Cancer - Diagnosis (2012) Cancer. Net, USA.

45. Human papilloma virus (HPV) vaccines (2016) National Cancer Institute."Risk Factors", USA.

46. Cervical Cancer (2016) Cancer Research Institute, USA.

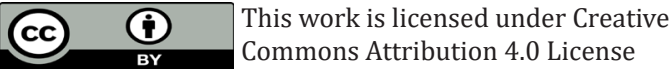

To Submit Your Article Click Here: Submit Article

DOI: $10.32474 /$ OAJBEB.2018.01.000106

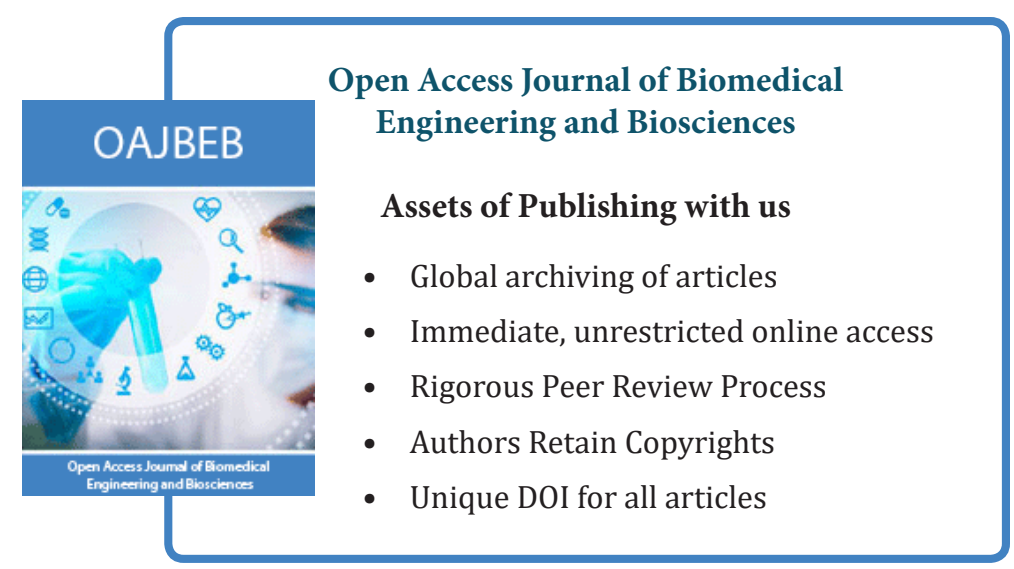

